\title{
$\bullet$ \\ Rhino-orbital Mucormycosis - A Dreaded Clinical Entity
}

\section{IJCRR}

Section: Healthcare

Sci. Journal Impact

Factor: 6.1 (2018)

ICV: 90.90 (2018)

(c) (i) (5)

Copyright@IJCRR

\section{Santosh Kumar Swain ${ }^{1}$, Smarita Lenka ${ }^{1}$, Saurjya Ranjan Das²}

'Department of Otorhinolaryngology, IMS and SUM hospital, Siksha "O" Anusandhan University, K8, Kalinganagar, Bhubaneswar-751003, Odisha, India; 'Department of Anatomy, IMS and SUM Hospital, Siksha O Anusandhan (Deemed to be University), Bhubaneswar-751003, Odisha, India.

\section{ABSTRACT}

Orbital mucormycosis is a filamentous fungal infection which is found to be inflaming the orbit along with the cerebrum, initiating from paranasal sinus, is scrutinized in the department of Otorhinolaryngology. Mucorales remains common and frequently isolated microorganisms, causing mucormycosis but Mucor spp. and Rhizomucor spp., are responsible for $70 \%$ of total cases. Inflammation is initiated at paranasal sinus and is disseminated to orbit. The whole inflammatory process goes through four stages of pathogenesis. Germination is supported by high glucose, low oxygen concentration, high iron levels and acidic medium and then germinates into hyphae. This analytical review has tried to drag attention upon complete systematic steps of orbital mucormycotic infection including physical, clinical as well as the pathological presentation of orbital mucormycosis. Rhino-orbital mucormycosis is a dreaded clinical entity. It invades and occludes vascular lumina, forms a characteristic black eschar on paranasal sinus. The disease, through the ethmoid and maxillary sinuses, spreads to the paranasal sinuses into the orbit and then to the cranial cavity by way of the orbital apex, cribriform plate, orbital nerves or orbital vessels. It could be diagnosed by histopathologically, magnetic resonance imaging (MRI) and computed tomography (CT) scan. Epithelial cells and phagocytic cells help in host defence mechanism but Mucorales spores trap the nasal epithelial cells coming to contact with GRP78 through fungal spore coat protein CotH3 and harm the primary cell lining. By utilizing iron chelators, hyperbaric oxygen and immunityboosting of individuals can be proved as a complementary process to the management of orbital mucormycosis.

Key Words: Mucormycosis,rhino-orbital mucormycosis, Amphotericin B. paranasal sinus

\section{INTRODUCTION}

Mucormycosis often found in immunocompromised patients, specifically in patients with diabetic ketoacidosis. Orbital mucormycosis is a fungal infection in orbit due to infectious fungal agents causing minor or major orbital infections. Fungal infection in orbit may lead harm to nearby tissues and further result in visual impairment and often death. Addition to this, fungal infection can invite many other types of infections along with other inflammations. Orbital mucormycosis is just like an opportunistic fungal infection, also known as phycomycosis or zygomycosis. Mucormycosis is named so because organisms causing mucormycosis belong to mucoraceae family. As the fungus is spore-forming in nature they could be inhaled through nose and mouth on a very regular basis by humans. These are widespread and ubiquitous, found in decaying vegetables and fruits, in soil, air, animal faeces and remaining food items. ${ }^{1}$ The most common orbital fungal disease is mucormycosis. Swelling in eyes, deadness in the face are the commonest symptoms of orbital mucormycosis. The orbital wall gets inflamed and marked as an infectious thickening, expansion in the orbital wall, orbital fat and extraocular muscles. Patients having poorly controlled diabetes mellitus and lower immunity are prone to this fungal attack. In case of orbital mucormycosis, the infection starts through the nose and paranasal sinus usually remained as initial as well as a primary site of the fungal entry point, and invasion to orbit always possess secondary involvement eventually through intracranial structures via orbital apex or through blood vessels. ${ }^{2}$ In this review the guideline is proposed for both sinonasal as well as orbital mucormycosis as sinus acts as the initial infection site of orbital mucormycosis but orbital mucormycosis is the focusing term of this review as the orbit is affected in all kind of incorporation of fungal infection including invasive and non-invasive forms.

\section{Corresponding Author:}

Dr. Santosh Kumar Swain, Professor, Department of Otorhinolaryngology, IMS \& SUM Hospital, Siksha "O" Anusandhan University, K8, Kalinganagar, Bhubaneswar-751003, Odisha, India; Cell: +91-9556524887; Email: santoshswain@soa.ac.in

ISSN: 2231-2196 (Print)

Received: 22.07 .2020
ISSN: $0975-5241$ (Online)

Revised: 05.10 .2020
Accepted: 23.10 .2020
Published: 14.12 .2020 


\section{METHODOLOGY}

Research articles regarding orbital mucormycosis were searched through a multiple approaches. First, we conducted an online search of the PubMed, Scopus, Google Scholar and Medline database with the word orbital mucormycosis including its epidemiology, etiopathology, clinical presentations, investigations and current treatment. The abstracts of the published article were identified by this search method and other articles were identified manually from the citations. This review article reviews the epidemiology, etiopathology, clinical presentations, investigations and current treatment of orbital mucormycosis This review article presents a baseline from where further prospective trials can be designed and help as a spur for further research in this clinical entity where not many studies are done. The objective of this review article is to provide precise and brief knowledge regarding the prevention and treatment of the orbital mucormycosis. This article is specially focused on managing a dreaded clinical entity such as orbital mucormycosis.

\section{EPIDEMIOLOGY}

Mucormycosis is an invasive fungal infection which has been observed throughout the world with its severity. Mostly on tropical and subtropical countries like India are ideal for fungal growth during the transition from summer to rainy season between August and November. Due to delay in the diagnostic process orbital mucormycosis has a high mortality rate of $25 \%-62 \%$ than recovery. ${ }^{3}$ Rising cases of mucormycosis remain high in China and India than other countries among uncontrolled diabetic patients but another survey reported that incidence of mucormycosis is higher with Europe on top with (34\%) and followed by Asia with (31\%), South America (28\%), Africa (3\%), Australia and New Zealand $(3 \%){ }^{4}$

\section{ETIOPATHOLOGY}

Mucormycosis is caused by the saprophytic fungi of the order Mucorales. Rhizopus, Rhizomucor and Absidia are the commonly isolated organisms from the patients of orbital mucormycosis. ${ }^{5}$ About $70 \%$ of the rhino-orbital mucormycosis are found in diabetic patients with ketoacidosis and other risk factors are patients with hematopoietic stem cell or solid organ transplantation. ${ }^{6}$ Mucormycosis is a fungal infectious disease and is life-threatening inflammation seen in immunocompromised patients in the result of either diabetes ketoacidosis; when sugar level of blood is increased and acidic substances called ketones accumulate to alarming levels in the body, neutropenia; in presence of abnormally few neutrophils in the blood, leading to increased susceptibility to infection, increased serum levels of available iron, or organ transplantation surgery that leads to lacking enough red blood cells in the body. ${ }^{7}$ The kingdom fungi classified into four phylums; Ascormucota, Basidiomycota, Zygomycota and Deuteromyces. Zygomycetes came under phylum Zygomycota and Mucorales came underclass Zygomycetes. Mucorceae family comes under order Mucorales (Figure 1). Thus disease caused by mucor is identified as mucormycosis. ${ }^{8}$ Mucormycosis is generated by fungi belonging to the class of zygomycetes and to the order of Mucorales, which also has species like Absidia, Apophysomyces, Mucor, Rhizomucor, Rhizopus, Cunninghamella and Saksenaea. The most common isolated and etiological factor of mucormycosis is Rhizopus spp., Mucor spp. and Rhizomucor spp., responsible for $70 \%$ of total cases. ${ }^{9}$ Mucormycosis is a systemic infection that can also affect lungs, kidney, bone, bladder and gastrointestinal tract, skin, heart and brain etc through contaminated tongue depressors. ${ }^{10}$

Mucormycosis is particularly seen in uncontrolled diabetes mellitus persons but also could be of high risk in case of immune-compromised hosts, patients with hepatitis, lymphoma, leukaemia, multiple myeloma, septicaemia, renal failure, cirrhosis, patients on steroids intravenous or chemotherapy and drug abusers. Spores produced by Mucorales inhaled by the host from the environment through the nose. Emerging from paranasal sinus and nasal cavity colonisation, mucormycosis infection spreads to the orbits after spreading to the palate and the pharynx..$^{11}$ The term rhino-orbital mucormycosis refers to the entire spectrum of the disease as due to proximity it affects nasal tissue to orbit and get vigorous at affecting the central nervous system. It usually starts in stage I, the sino-nasal tissues (limited sino-nasal disease), advances to the orbits (limited rhino-orbital disease) could be stage II infection and lastly affects the central nervous system (rhinoorbital-cerebral disease) referred as stage III of infection. ${ }^{12}$ Pathogenesis includes microbial infection, inflammation, malignancy and tissue breakdown to cause disease, a pathogen must successfully achieve four steps or stages (Figure 2) of pathogenesis: exposure (contact), adhesion (colonization), invasion, and infection. ${ }^{13}$ Orbit act as the site for mucormycosis which is initiated particularly in case of immunesuppression or having an immune deficiency in the body. Mucormycosis seeks sufficient iron from the host for better growth. A healthy host defends against mucormycosis by the process of sequestration of iron in the serum through specialized iron-binding protein. Regulatory mechanism of phagocytic cells, circulation of neutrophils, tissue macrophages, and endothelial cells block the formation of infection in the tissue and upcoming endovascular invasion. But as of vulnerable hosts, a defence mechanism of the body is disturbed. Dissociation of free iron from sequencing protein occurred by the acidic $\mathrm{pH}$ of the serum helps for releasing of free iron leads to lowering phagocytic defence mechanism and allows rapid fungal growth. This situation invites deficiency 
in cell number, acidosis, hyperglycaemia and finally damage endothelial cells by fungus causes subsequent thrombosis, tissue necrosis and spreading of the fungal infection. Haematological malignancies are a crucial etiological threat for mucormycosis. ${ }^{14,15}$

\section{HOST DEFENCE MECHANISM DURING MUCO- RALES INVASION}

Patients of rhino-orbital mucormycosis are often with poor immunity and diabetes ketoacidosis. During initial incubation of Mucorales, both types of phagocytes such as polymorphonuclear and mononuclear phagocytes of normal hosts engulf Mucorales by generating cationic peptides and oxidative metabolites defensins. Specialized iron-binding proteins, neutrophils and tissue macrophages, endothelial cells are the concerned host defence mechanisms that prevent the establishment of infection. ${ }^{16}$ When spore entered on paranasal sinus, the epithelial cell is affected initially as it acts as the primary barrier of all type of infection. Acidic conditions and high-glucose, through the interaction of the fungal ligand, can occupy human umbilical vein endothelial cells. Mucorales ligands can recognize host receptors which are specific to individual cell types of infected site and forms host-ligand interaction. Some host factors can promote the fungal ligand-host receptor interaction that eventually leading to the fungal attack on the epithelial cell of respective host niches. Almost $40 \%$ of invasion results within the first three hours of fungal inoculation. Within 30 to 40 hours of inoculation, about 40 to $80 \%$ of nasal epithelium got infected. ${ }^{17}$ As discussed previously, the rising concentration of ketone, iron and glucose level in diabetes ketoacidosis bodies trigger the nasal glucose-regulated protein 78 (GRP78) expression during adverse condition like a fungal attack. Mucorales spores trapped the GRP78 interacted nasal epithelial cells on there to infect and damage the primary cell lining through fungal spore coat protein $\mathrm{CotH} 3$ (Figure 3).$^{18}$ Specific bond formation of Mucorales with a single nasal epithelial cell protein are resulted by incubation of Mucorales of the nasal epithelial cell with extracts of biotin-labelled total protein. Nasal epithelial cell membrane proteins with antiGRP78 polyclonal antibody, isolated through SDS-PAGE gel containing anti-biotin antibodies immunoblot, seen as 78 $\mathrm{kDa}$ band that bound to that organism responsible for epithelial cell damage can be confirmed. ${ }^{19}$ Polyclonal antibodies against either GRP78 or CotH3 can prevent damage of nasal epithelial cells by abolishing Mucorales invasion. ${ }^{17,20}$ Mucorales hyphal damage is minimised by Human polymorphonuclear neutrophils (HPMNs) associated with impaired oxide ion $\left(\mathrm{O}_{2}^{-}\right)$. Due to rapid induction of Nuclear factorkappa B (NF-кB) pathway-related genes and enhancement of response by a stimulus in tolls like receptor 2 mRNA, exposure of Mucorales hyohae and human polymorphonuclear neutrophils (HPMNs) results. ${ }^{21} \mathrm{NF}-\kappa \mathrm{B}$ is a major transcription factor that helps to regulate genes behind adaptive and innate immune response and activated when either B- or Tcell receptor is triggered through some of the distinct signalling components.

\section{ROLE OF IRON UPTAKE IN ORBITAL MUCOR- MYCOSIS}

As previously mentioned that heavy iron intake through blood or serum could be a critical factor for induction of mucormycosis. The sequestration of iron with host carrier protein-like transferrin, ferritin, and lactoferrin interaction can avoid the toxicity of free iron and limits high iron availability and this strategy can be used as a universal host defence mechanism against Mucorales. ${ }^{21}$ Diabetic ketoacidosis supports increasing of iron in the body and this simulated acidotic condition decreases the iron-transfer in binding capacity and disrupts defence mechanism of the host. ${ }^{22}$ With the help of using low-molecular-weight iron chelators or high-affinity iron permeases, Mucorales can obtain iron and reduced to ferric into soluble ferrous form by surface reductase. Then that reduced ferrous captured by a protein complex, this undergoes genome sequencing and identify 1 high-affinity iron permease (FTR1), 6 copper oxidases, and 3 ferric reductases. Gene encodes high-affinity iron permease expressed by $\mathrm{Mu}-$ corales. Then the reduction of FTR1 or suppression of FTR1 assertion by RNA-1 reduces the virulence of mucormycosis (Figure 4). ${ }^{23}$

\section{CLINICAL PRESENTATIONS}

Orbital mucormycosis is a true fungal pathogenic and opportunistic infection. Initial symptoms resulting from sinus spread to orbit may develop orbital apex syndrome at that condition. The patient often presents with facial or eye pain and numbness on the facial area. If it is untreated, results in proptosis, chemosis and ptosis. Sometimes the rhino-orbital mucormycosis is associated with involvement of the cavernous sinus and affects III, IV, V, VI cranial nerves and cause paralysis of these nerves and also blindness. ${ }^{24}$ If the cavernous sinus is affected, it leads to paralysis of the III, IV, V, VI cranial nerves and also cause blindness. ${ }^{3}$ Vulnerable patients are infected by inhaling spores into the oral and nasal mucosa which grow and spread into the tissue using fungal hyphae. The hyphae are 5 to 15 microns wide, have rare septations and are irregularly branched. Here germination is supported by high glucose, acidic medium and high iron levels, low oxygen concentration and germinates into hyphae during stage I orbital mucormycosis. ${ }^{16}$ During stage II organisms developing orbital mucormycosis has a propensity to invade and occlude vascular lumina, leading to form a characteristic black 
eschar or lession. The disease process then spreads through the ethmoid and maxillary sinuses to the paranasal sinuses that reach into the orbit. The infection will move deeper into the cranial cavity through the cribriform plate, orbital apex, orbital nerves (perineural) or orbital vessels (peri or intravascular). ${ }^{25}$ Various elements lead the orbit to infection due to its inferior, middle, and superior relationship to the paranasal sinuses; a paper-thin bone of the lamina papyracea separates the orbit from the ethmoid sinuses and the direct spread of infectious organisms which permits due to lack of valves in the inferior and superior ophthalmic veins in the midface. ${ }^{26}$

Black eschar formation by inoculation of fungal spores through sinus, spread to intracranial cavity may be rapid and devastating, leading to a fatal outcome. Due to the absence of sinus disease and naso-canthal eschar, direct extension after periorbital cutaneous inoculation may result in secondary orbital mucormycosis. In association with the marked orbital inflammatory process in a patient with mucormycosis optic nerve infarction developed within two weeks following the presentation and optic nerve thickening and infarction is the first and only sign of orbital involvement. ${ }^{27}$ The early clinical presentations of orbital involvement include eye pain, proptosis and defective acuity of the vision. The orbital involvement is associated with a high density of the soft tissue and engorgement of the optic nerve. Clinically it is characterised frequently as fever, sinusitis, headache, periorbital or facial swelling, ptosis, visual loss and ophthalmoplegia. It also represents Cranial nerve palsies, proptosis and epistaxis reportedly. ${ }^{28,29}$ Along with the immunocompromised state, severe trauma, cancers, major burns, AIDS, bone marrow stem cell transplants, iron levels as well as an overload of aluminium may be the risk factors for lowering of immunity and initialization of mucormycosis. Regular dialysis treatments, deferoxamine therapy, cirrhosis of the liver and hepatic failure, infant acidosis following vomiting and diarrhoea, intravenous drug abuse and protein-calorie malnutrition are clinical symptoms and also risk factors for immunity failure.

Symptoms like fever, rhinitis, sinusitis, headache, facial pain with swelling, nasal ulceration, granular or purulent nasal discharge, hemiplegia or stroke, epistaxis and cognitive disorder can be primarily seen. Orbital mucormycosis patients show signs of orbital pain, facial hypoaesthesia and abrupt vision loss. The signs also include ophthalmoplegia, chemosis, hypoaesthesia, proptosis, ptosis and periorbital cellulitis. Various positions of the face like eyelid that is near to the maxillary sinus or the nose may be violaceous, erythematous or lead to cell death at the end. Dried blood resembling black necrotic tissue or a purulo-sanguineous exudate with thin, pink, and watery presentation and with an uncomfortable smell may be detected on the nasal septum, turbinates and palate. The bone and cartilage may become soft with further perforation with thick grey, green or yellow purulent drainage as the affected area share the ischemic necrosis. ${ }^{8,30}$ The prognosis of the rhino-orbital mucormycosis worsens with it involves cavernous sinus.

\section{DIAGNOSIS}

Diagnosing of the orbital mucormycosis requires histopathological evidence of fungal invasion of the tissues and the extent of involvement can be evaluated with CT or MRI scans. ${ }^{31}$ Along with above-mentioned symptoms like the history of hypothyroidism, chronic otitis media, diabetes mellitus, obstructive sleep apnea and recurrent vertigo also remain as regular signs that mostly effecting orbital mucormycosis. Despite, nasal entry point act as the initiation of orbital mucormycosis, nasal swab culture for fungal identification may not be sufficient for better evaluation. If there are any changes seen in normal appearance in the nasal mucosa then an early and primary infection of orbital mucormycotic infection can be detected with thorough endoscopy of nasal with an eye of suspicion. During the inspection the common site of mucormycosis should be middle turbinate, septum, palate and inferior turbinate. Presence of sensation and decreased bleeding in these sites could be the primary diagnosis of orbital mucormycosis. ${ }^{32}$

The histopathological diagnostic method can be chosen in the purpose of examining tissue of infected area under a microscope. It can detect the actual assimilation stage of infection. Dextrose Sabouraud's Agar medium is mostly applicable for fungal growth following a period of growth with appropriate time and temperature. Hematoxylin and Eosin stain helps to detect aseptate broad fungal hyphae and shows cytoplasm stained pink-orange and nuclei stained with dark blue or purple. Lactophenol cotton blue staining is able to detect mucor sporangium with sporangiophore as high concentration of the phenol present in lactophenol deactivates lytic cellular enzymes thus the cells do not lyse. ${ }^{33}$ Periodic acid-Schiff (PAS) is a staining method used to detect polysaccharides such as glycogen, and mucosubstances such as glycoproteins, glycolipids, and mucins in examining tissues. Tissue examination using PAS staining can validate fungal hyphae with right-angle branching. ${ }^{34}$ The CT scan is a very successful diagnostic method of orbital mucormycosis where both sphenoid sinuses are involved, as revealed by the paranasal sinus. The patient who goes through transnasal navigated endonasal debridement, along with adjacent soft tissue of ethmoid and sphenoid sinus mucosa can also be diagnosed through CT scan. The MRI scan can detect fungal involvement of the orbital apex and brain abscess both before and after clinical examination, bilateral preseptal soft tissue swelling, soft tissue swelling in the right lateral and superior orbit and ill-defined enhancement of orbital apex extending interiorly with the optic nerve. ${ }^{35,36}$ Another diagnostic method may be used in case of no evidence of sinus and brain abnormalities, and no fluid col- 
lections; magnetic resonance venography in which a combination of a computer, radiofrequency and a large magnet is applicable for analysing proper images of structures and organs within the orbit. Intravenous (IV) contrast dye and Magnetic resonance technology are used in this procedure for clear visualisation of veins and the right cavernous sinus observes decreased flow. ${ }^{36}$

\section{TREATMENT}

There is a treatment for orbital mucormycosis include aggressive surgical debridement, intravenous liposomal amphotericin-B and hyperbaric oxygen therapy. Orbital mucormycosis is completely an immunocompetent disease, mostly affecting immunocompromised patients. Therefore immunity-boosting of a Mucormycosis patient can be proved as better management of this disease. Incorporation of granulocyte-macrophage colony-stimulating factor and gamma interferon to the systematic plan could become favourable management to leukemic patients with orbital mucormycosis because cytokines activate host phagocytic activity including granulocyte-macrophage colony-stimulating factor, gamma interferon that is able to kill Mucorales in vitro. ${ }^{37}$ Utilization of iron chelators allow entry of Mucorales into the host but some chelators such as deferoxamine and deferasirox restricts allow of Mucorales invasion, maintain the balance of iron level and managed mucormycosis pathogenesis. ${ }^{12,34}$ Another therapy suggested that the amount of oxygen in blood increases by hyperbaric oxygen. Normal levels of blood gases and tissue function is restored by the increase in blood oxygen temporarily to fight against infection and promote healing. ${ }^{35,38}$ The ability of neutrophils is improved by high oxygen pressure to kill infected organisms and hamper germination of fungal spore and mycelia. ${ }^{14}$ Control of the system administration and insertion with local antifungal therapy, aggressive surgical debridement, predisposing factors and maintaining of self-hygiene could be implemented as the best way of management to this disease. Proper balancing activity like the use of immunosuppressive drugs, control of underlying hematologic malignancies and control of diabetes can suppress the rate of complications at the initial stage of orbital mucormycosis. Proper ways of management should be implemented in our daily lifestyle as it becomes very important to maintain self-hygiene to avoid infection. But in case of a susceptible patient proper management should be introduced before surgical procedure.,39 Collecting summaries of many case studies it is reported that Amphotericin B, Posaconazole and liposomal Amphotericin B has successful outcomes towards management and treatment of orbital mucormycosis as these therapies allow best tissue penetration of the drug. ${ }^{40,41}$

\section{CONCLUSION}

Orbital mucormycosis has the propensity to invade and causing serious threats by hampering the host immunity both in normal and having diabetes ketoacidosis patients. Self-hygiene, adopting proper care and several applications to boost host immunity can terminate the entry of fungal spores and alternately can eradicate of orbital mucormycosis. Early detection and well prognosis are mutual necessary processes for orbital mucormycosis. Following disease management, the appropriate process of diagnostic method and by controlling predisposing factors, orbital mucormycosis can come under control. Before surgical process, proper progression rate should be known clearly for getting better surgical results. Availability of best disease management by using successful treatment therapy can show good outcomes towards the change of empirical use of antibiotics and drugs against orbital mucormycosis.

\section{ACKNOWLEDGMENT}

Authors acknowledge the immense help received from the scholars whose articles are cited and included in references to this manuscript. The authors are also grateful to authors / editors / publishers of all those articles, journals, and books from which the literature for this article has been reviewed and discussed.

\section{Conflict of Interest: Nil}

Source of Funding: Nil

\section{REFERENCES}

1. Shamanna K, Fathima A, Sowjanya S. Rhino-Orbito-Cerebral Mucormycosis: Our Experience. Headache 2019;15:75.

2. Gavito-Higuera J, Mullins CB, Ramos-Duran L, Sandoval H, Akle N, Figueroa R. Sinonasal fungal infections and complications: a pictorial review. J Clin Imaging Sci 2016;6:23.

3. Swain SK, Sahu MC, Baisakh MR. Mucormycosis of the Head and Neck. Apollo Med 2018;15:6.

4. Jeong W, Keighley C, Wolfe R, Lee WL, Slavin MA, Kong DC, et al. The epidemiology and clinical manifestations of mucormycosis: a systematic review and meta-analysis of case reports. Clin Microbiol Infect. 2019;25:26-34.

5. Swain SK, Behera IC, Mohanty JN. Mucormycosis in head-andneck region-Our experiences at a tertiary care teaching hospital of Eastern India. Annl Indian Acad Otorhinolaryngol Head Neck Surg 2019;3(2):58.

6. Sarvestani A, Pishdad G, Bolandparvaz S. Predisposing Factors for Mucormycosis in Patients with Diabetes Mellitus; An Experience of 21 Years in Southern Iran. Bull Emerg Trauma 2013;1(4):164-70.

7. Ibrahim AS, Spellberg B, Walsh TJ, Kontoyiannis DP. Pathogenesis of mucormycosis. Clin Infect Dis 2012;54:S16-22.

8. Kirszrot J, Rubin PA. Invasive fungal infections of the orbit. Int Ophthalmol Clin 2007;47:117-32. 
9. Roden MM, Zaoutis TE, Buchanan WL, Knudsen TA, Sarkisova TA, Schaufele RL, et al. Epidemiology and outcome of zygomycosis: a review of 929 reported cases. Clin Infect Dis 2005;41:634-53.

10. Wali U, Balkhair A, Al-Mujaini A. Cerebro-rhino orbital mucormycosis: an update. J Infect Public Health 2012;5:116-26.

11. Hosseini SM, Borghei P. Rhinocerebral mucormycosis: pathways of spread. Eur Arch Otorhinolaryngol 2005;262:932-8.

12. Nithyanandam S, Jacob MS, Battu RR, Thomas RK, Correa MA, D'Souza O. Rhino-orbito-cerebral mucormycosis. A retrospective analysis of clinical features and treatment outcomes. Indian J Ophthalmol 2003;51:231-6.

13. Swain SK, Debta P, Bishoyi AK, Padhy R. Invasive aspergillosis of tympanomastoid cavity. J Scient Soc 2019;46(3):106.

14. Spellberg B, Edwards J Jr, Ibrahim A. Novel perspectives on mucormycosis: pathophysiology, presentation, and management. Clin Microbiol Rev 2005;18:556-69.

15. Abedi E, Sismanis A, Choi K, Pastore P. Twenty-Five years experience treating Cerebro-Rhino-Orbital mucormycosis. Laryngoscopw 1984;94:1060-2.

16. Ananthaneni AR, Undavalli SB, Velagapudi RP, Guduru VS. Mucormycosis: an atrocious mate of patients with diabetes. BMJ Case Rep 2013;2013:bcr2013009600.

17. Alqarihi A, Gebremariam T, Gu Y, Swidergall M, Alkhazraji S, Soliman SS, et al. GRP78 and Integrins Play Different Roles in Host Cell Invasion During Mucormycosis. Mbio 2020;11(3):118.

18. Watkins TN, Gebremariam T, Swidergall M, Shetty AC, Graf $\mathrm{KT}$, Alqarihi A, et al. Inhibition of EGFR signaling protects from mucormycosis. MBio 2018 5;9(4):1-13.

19. Isberg RR, Leong JM. Multiple $\beta 1$ chain integrins are receptors for invasin, a protein that promotes bacterial penetration into mammalian cells. Cell 1990;60:861-71.

20. Liu M, Spellberg B, Phan QT, Fu Y, Lee AS, Edwards JE, et al. The endothelial cell receptor GRP78 is required for mucormycosis pathogenesis in diabetic mice. J Clin Invest 2010;120:191424.

21. Chamilos G, Lewis RE, Lamaris G, Walsh TJ, Kontoyiannis DP. Zygomycetes hyphae trigger an early, robust proinflammatory response in human polymorphonuclear neutrophils through tolllike receptor 2 induction but display relative resistance to oxidative damage. Antimicrob Agents Chemother 2008;52:722-4.

22. Artis WM, Fountain JA, Delcher HK, Jones HE. A mechanism of susceptibility to mucormycosis in diabetic ketoacidosis transferrin and iron availability. Diabetes 1982;31:1109-14.

23. Ibrahim AS, Spellberg B, Walsh TJ, Kontoyiannis DP. Pathogenesis of mucormycosis. Clin Infect Dis 2012;54:S16-22.

24. Sarvestani A, Pishdad G, Bolandparvaz S. Epidemiology and Clinical Characteristics of Mucormycosis in Patients with Leukemia; A 21-year Experience from Southern Iran. Bull Emerg Trauma 2014;2(1):38-43.

25. Bhatti MT. Orbital syndromes. Semin Neurol 2007;27:269-87.

26. Sharma S, Jakati S. Sino-Orbital Invasive Fungal Infections in
Immunocompetent Hosts. Curr Fungal Infect Rep 2020;14:24651.

27. Mathur S, Karimi A, Mafee MF. Acute optic nerve infarction demonstrated by diffusion-weighted imaging in a case of rhinocerebral mucormycosis. AJNR Am J Neuroradiol 2007;28:489-90.

28. Hadzri MH, Azarisman SM, Fauzi AR, Kahairi A. Invasive rhinocerebral mucormycosis with orbital extension in poorlycontrolled diabetes mellitus. Singapore Med J 2009;50:107-9.

29. Prakash MV, Kumar PA, Umamaheswari TG, Harivanzan V. The clinical pattern of orbital mucormycosis in a tertiary eye care hospital. TNOA J Ophthalmic Sci Res 2020;58:14-6.

30. Luo QL, Orcutt JC, Seifter LS. Orbital mucormycosis with retinal and ciliary artery occlusions. Br J Ophthalmol 1989;73:6803.

31. Swain SK, Sahu MC, Banerjee A. Non-sinonasal isolated facio-orbital mucormycosis-A case report. J Mycologie Med 2018;28(3):538-41.

32. Galletti B, Freni F, Meduri A, Oliverio GW, Signorino GA, Perroni $\mathrm{P}$, et al. Rhino-Orbito-Cerebral Mucormycosis in Diabetic Disease Mucormycosis in Diabetic Disease. J Craniofac Surg 2020;31:e321-4.

33. Guarner J, Brandt ME. Histopathologic diagnosis of fungal infections in the 21st century. Clin Microbiol Rev 2011;24:247-80.

34. Singh VP, Bansal C, Kaintura M. Sinonasal Mucormycosis: A to Z. Indian J Otolaryngol Head Neck Surg 2019;71:1962-71.

35. Arndt S, Aschendorff A, Echternach M, Daemmrich TD, Maier W. Rhino-orbital-cerebral mucormycosis and aspergillosis: differential diagnosis and treatment. Eur Arch Otorhinolaryngol 2009;266:71-6.

36. Chahal HS, Abgaryan N, Lakshminarayanan R, Glover AT. Orbital mucormycosis following periorbital cutaneous infection. Ophthalmic Plast Reconstr Surg 2017;33:S146-8.

37. Gil-Lamaignere C, Simitsopoulou M, Roilides E, Maloukou A, Winn RM, Walsh TJ. Interferon-gamma and granulocytemacrophage colony-stimulating factor augment the activity of polymorphonuclear leukocytes against medically important zygomycetes. J Infect Dis 2005;191:1180-7.

38. Soman R, Sunavala A. Management of Invasive Fungal Sinusitis. In: Mankekar G, editor. Invasive Fungal Rhinosinusitis. New Delhi:Springer India;2014.p.63-75.

39. Swain SK, Sahu MC, Debdta P, Baisakh MR. Primary fungal laryngitis: An overlooked clinical entity. Apollo Med 2019;16(1):11.

40. García-Covarrubias L, Barratt DM, Bartlett R, Van Meter K. Tratamiento adjunto con oxigenación hiperbárica en mucormicosis. Presentación de cinco casos tratados en la misma Institución y revisión de la literatura. Rev Invest Clín 2004;56:515.

41. Mohamed MS, Abdel-Motaleb HY, Mobarak FA. Management of rhino-orbital mucormycosis. Saudi Med J. 2015;36:865-8. 


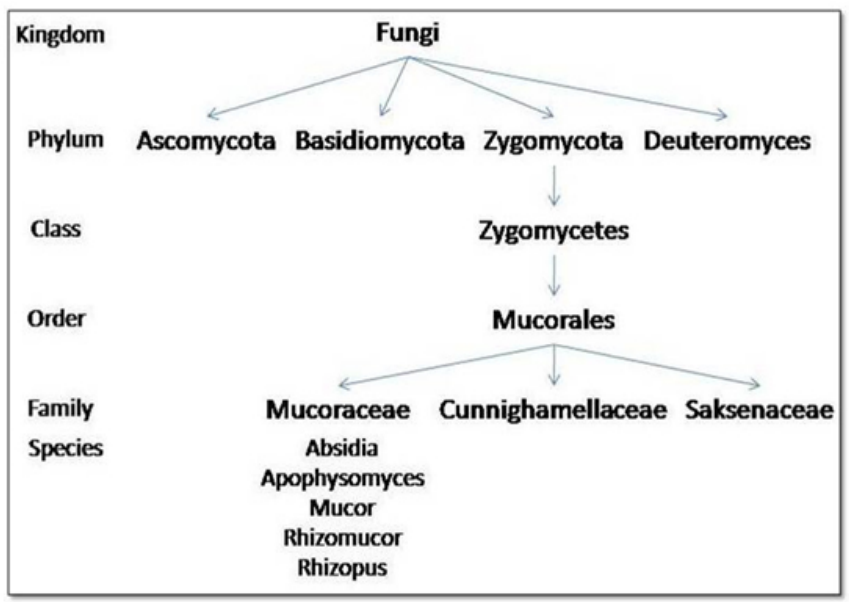

Figure 1: Classification of fungi defining Mucoraceae family.

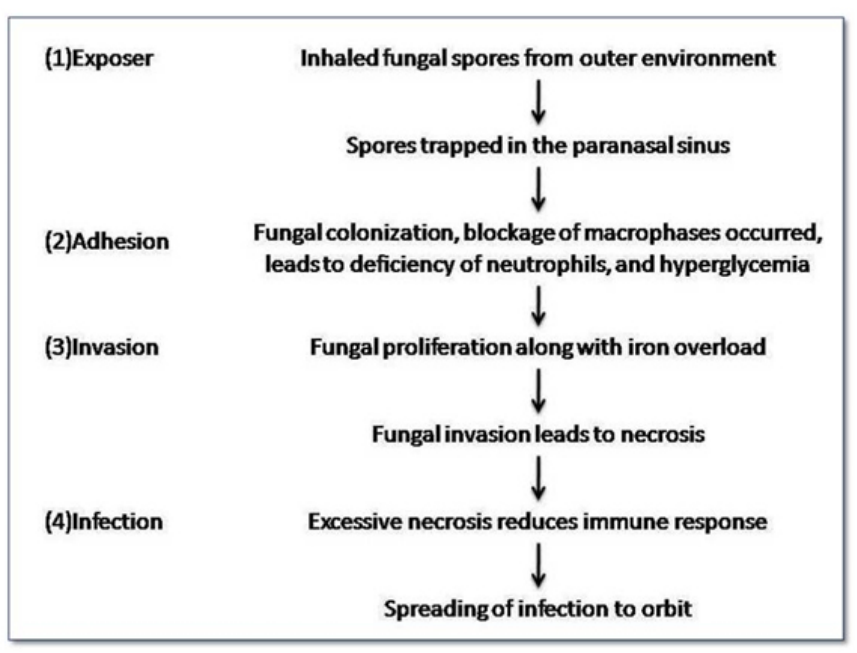

Figure 2: Pathogenesis of orbital mucormycosis.

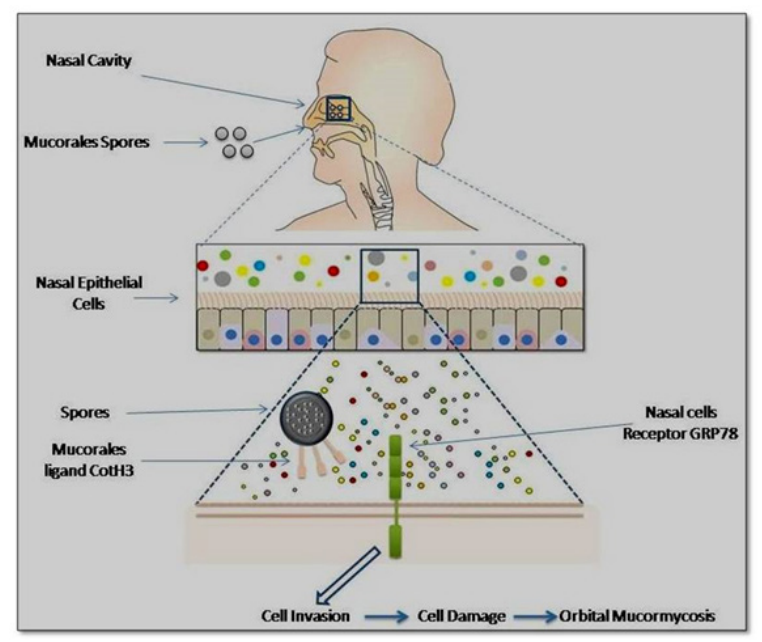

Figure 3: Entry of Mucorales spores and epithelial cell invasion causing orbital Mucormycosis.

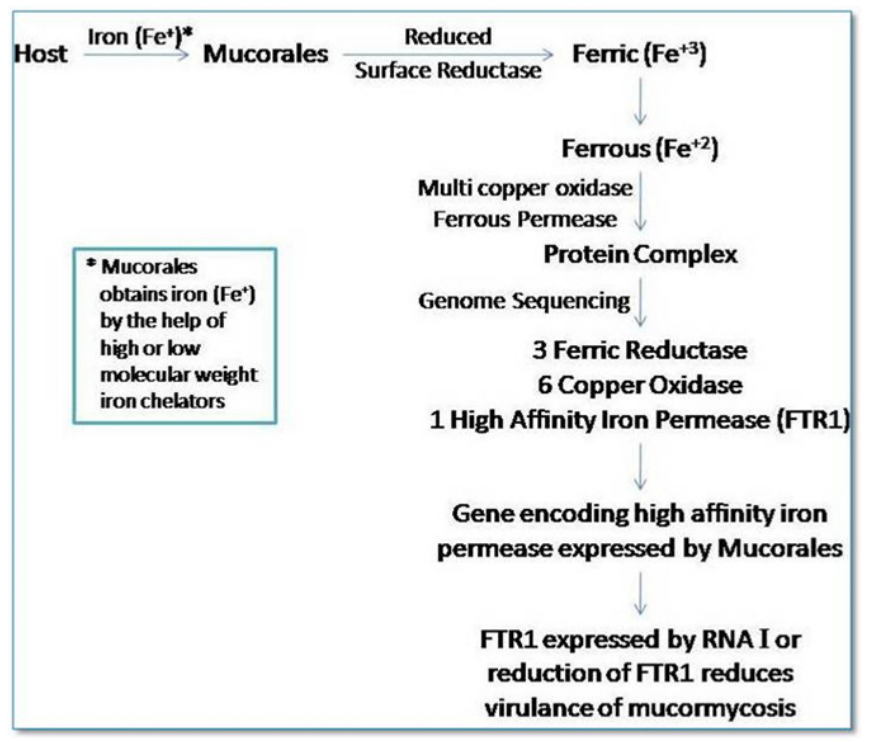

Figure 4: Role of Iron chelators reducing virulence of mucormycosis. 